UDC 535.37

\author{
D. Sokolov ${ }^{*}$ K. Katpaeva, V.Kurnosov \\ IETP, Departamentof physics and technology, al-Farabi Kazakh National University, \\ al-Farabi 71, Almaty 050012, Kazakhstan; \\ *e-mail: Dmitriy.Sokolov@kaznu.kz
}

\title{
Investigation of cryoemission characteristics during ethanol condensation
}

\begin{abstract}
Physics of cryocondensates growth and properties is one of the research areas in which scientists deal with real and often non-equilibrium states, which represent a purely scientific and practical interest. In this case, there is a very limited list of factors that determine the growth process and morphology (structure) of received and test substances.This primarily refers to the thermodynamic parameters of cryocondensation - gas phase pressure and substrate temperature. The substrate material and its morphology can play a part at the initial stage of phase formation in the study of epitaxial layers in real cryocrystals properties formation, which is caused by an interaction between the gas particles and the substrate material, i.e. the adsorption nature of the earliest layers of condensate.

Experiments series of detailed study of the cryoemission phenomenon, which was detected during the condensation of nitrous oxide and ethanol onto a cold substrate was carried out.The uniqueness of this phenomenon implies the fact that at the current time there is no theoretical determination of this radiation origin. The study of this phenomenon has revealed a number of characteristics that are related to both the thermodynamic parameters of the condensation and the actual radiation. Ethanol accompanied by radiation, when the substrate temperature is $\mathrm{T}=16-60 \mathrm{~K}$. On the other hand, when the condensing pressure is above $10^{-3} \mathrm{Torr}$, radiation intensity increases. Furthermore, an optimal value of the gas phase pressure, in which the radiation intensity is maximum, exists for each of the condensation temperature in the range of $16-130 \mathrm{~K}$.

The main objectives of these studies are checking of a wider range of substances for their ability to cryocondensation radiation; study of the nature of radiation and its relaxation characteristics; study of thermally stimulated processes in condensed samples.

Key words: SCL - supercooled liquid, crystal plastic, monoclinic crystal, glass transition temperature, IR spectrum, ethanol, cryocrystall, laser interferometer.
\end{abstract}

\section{Introduction}

Photoprocesses are various. They are photolysis with optical vibrational-rotational transitions, single-quantum and multiquantum, or one- and multiple-quantum electronic-vibrational-rotational (EVR) transitions between molecules and ions electronic states. These processes are accompanied by photon emission and induced by collisions of neutral and charged particles. In this chapter, we consider theoretical and practical aspects, which are related to obtained ethanol emission [1].

The reasons of information lack of these processes are theoretical and methodological complexities, which accompany their study. Photoprocesses are especially non-equilibrium reactions, and their quantitative characteristics (sections, quantum yields, constants) depend on the particles states, which are involved in the process. It is very difficult to measure their concentration without damage for their state.

Earlier we have conducted studies in which an interesting behavior of nitrous oxide was obtained in the process of the sample condensation and thermal cycling. During the studying of the dynamic characteristics of gas-solid phase transformations, it was found out that the condensation of a metal substrate is accompanied by light emission in visible spectrum.

In this part of the research, we report our results of the cryocondensation radiation, which is accompanying the condensation of some gases (Figure 1). The main objectives of this research were: (a) - to check a broader range of substances for their ability to cryocondensation radiation; (b) to study the nature of radiation and its relaxation 
characteristics; (c) - to study the thermally stimulated processes in condensed samples. The studies were carried out at the installation and following the procedure, which we have previously described in detail [4 - 5]. Measurement of the radiation intensity was carried with photomultiplier tubes P25a-SS-0-100 with a frequency of $10^{-5}$ measurements per second.

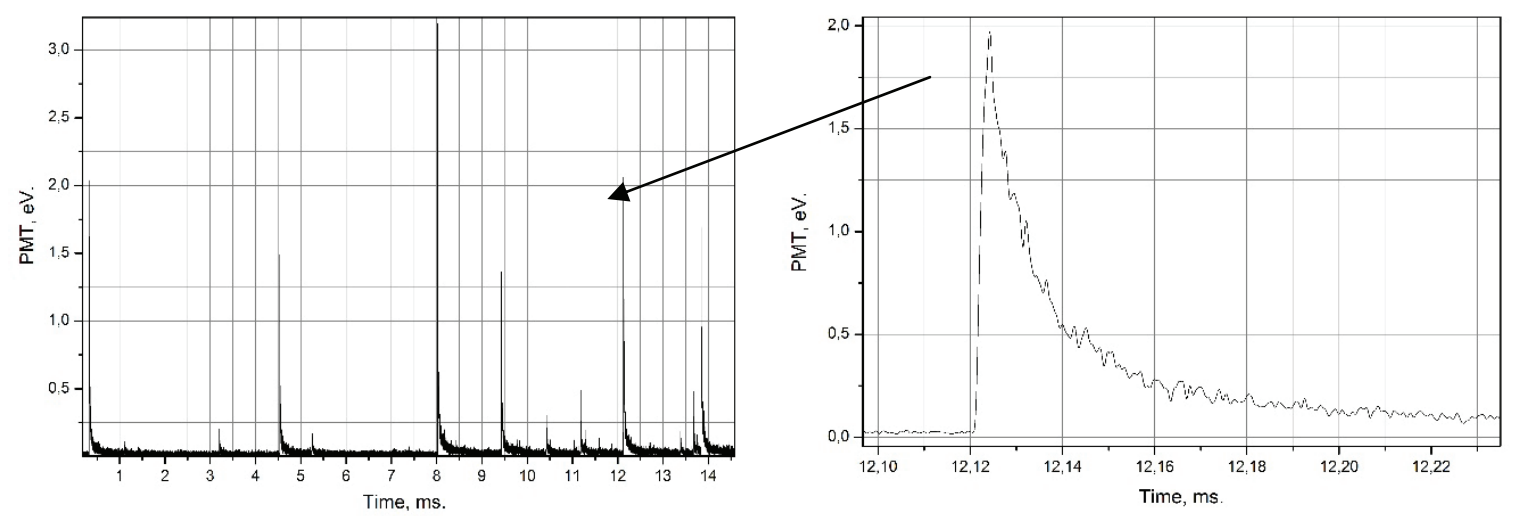

Figure 1 - Ethanol cryoemission during the condensation onto the cooled substrate at the temperature of $\mathrm{T}=16 \mathrm{~K}$.

When we were selecting the working substances, we proceeded from the assumption of the possible role of molecules intrinsic dipole moment in the creation of conditions for the cryoemission occurrence. Therefore we investtigated dipole molecules (nitrous oxide, water, ethanol), and molecules with zero intrinsic dipole moment (nitrogen, carbon dioxide and argon).

Thermal radiation - is electromagnetic radiation, which is produced due to the internal energy of the body. It is a continuous spectrum, the maximum of which depends on the body temperature. During the cooling, this maximum is shifting to longer wavelengths part of the spectrum [2].

Due to the fact, that atoms and molecules consist of charged particles, the substance emits electromagnetic waves. In particular, impulse excitation with following emission occurs when the atoms and molecules collide. A characteristic feature is that spectrum exponential obstruction begins when averaged emissivity of the Maxwellian distribution is above the hv kT energy. [3]

Radiation is called the equilibrium radiation if it is in thermodynamic equilibrium with a substance. The spectrum of this radiation is equivalent to the spectrum of a blackbody, and is described by Planck's law. Generally, however, the thermal radiation is not in thermodynamic equilibrium with the substance, thus the hotter body cools and cooler body heats. Kirchhoff's law determines the spectrum of this radiation.
The transitions without changing the spin quantum number, that is between states with the same multiplicity (singlet or triplet -cingletnye triplet), are allowed in electron spectroscopy. In consequence of the electronic transition the electron, spin does not change. Electronic transitions occur in $\sim 10^{-16} \mathrm{sec}$. Lifetime of the excited electronic states of atoms and molecules of $\sim 10^{-8}$ sec.

\section{Experimental results}

The conducted study observed effect revealed a number of characteristics related to both the thermodynamic parameters of the phase formation and the actual radiation.

Cryo condensation of ethanol is accompanied by radiation at substrate temperatures below 60 K.In slow annealing the first time after condensation observed flare.Figure 1 shows an example of the radiation of the ethanol condensation from the gas phase onto a cooled substrate at $16 \mathrm{~K}$ at the pressure of $\mathrm{P}=10^{-4}$ torr. To measure the radiation we used photomultiplier tube (PMT). A peak in a larger is represented on the right. In addition, single flares such as shown in Figure 2 are observed. Flares duration in most single is identical and is from 0.1 microsecond to 10 microseconds, depending on the value of intensity that can affect the direction of radiation to the receiver. 


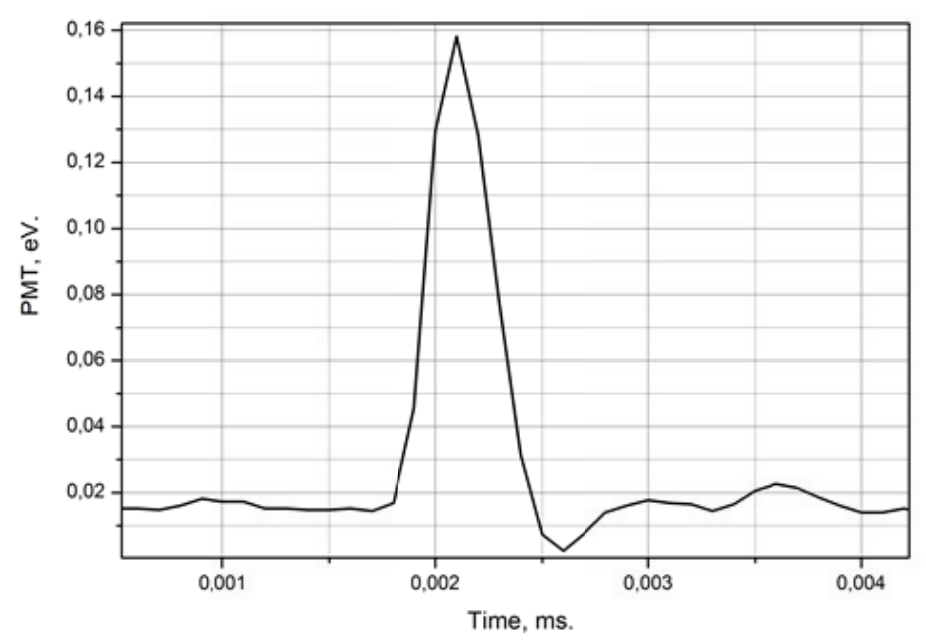

Figure 2 - Detected signal from the photomultiplier during condensation of ethanol to the substrate.

In the process of heating near the glass, transition temperature was also recorded radiation. This phenomenon requires further careful study, since at this stage, does not attempt to determine the wavelength of radiation that will help determine the radiation energy and understand what changes or transitions are responsible for this phenomenon.

From our data for $\mathrm{N} 2 \mathrm{O}$ and $\mathrm{C} 2 \mathrm{H} 5 \mathrm{OH}$ follows that there is a minimum value of the condensing pressure below which emission is absent. The value of this minimum condensing pressure at which the observed fluorescence is different for each of the investigated substances. Thus, for cry condensation of nitrous illumination starts at a pressure about $\mathrm{P}=10^{-5}$ Torr, whereas the value for $\mathrm{C} 2 \mathrm{H} 5 \mathrm{OH}$ is $\mathrm{P}=10^{-2}$ torr. Importantly, the cry condensation emission with $\mathrm{N} 2 \mathrm{O}$ $\mathrm{C} 2 \mathrm{H} 5 \mathrm{OH}$ visually observable with the naked eye.

\section{References}

1. PravilovA. M. Photo processes in molecular gases. - M.: Energoatomizdat, 1992. -352 p.

2. Elyashevich M.A. Thermal radiation // Physical encyclopedia / Ed. by ProkhorovA.M.M.: Soviet Encyclopedia. - P. 1988-1999.

3. Zasov A.V., Postnov K.A. The blackbody radiation // General Astrophysics. - Friazino, 2006. - Vol. 496 - P.32.

4. Drobyshev A., GarypoglyD. On some features of the cry condensation of nitrous oxide // Sov. J. Low Temp. Phys.-1990.- Vol. 16.-P. 936.

5. Drobyshev A., Aldiyarov A. et al. Transformation of cry vacuum condensates of ethanol near the glass transition temperature // Low Temp. Phys. - 2013. - Vol. 39.- P.460. 